Proceedings of the XIII National School of Superconductivity, Lạdek Zdrój 2007

\title{
Pseudogap-Like Phenomena in Cuprates as a Manifestation of Charge-Density Waves
}

\author{
T. Ekino ${ }^{a}$, A.M. Gabovich ${ }^{b}$, Mai Suan Li $^{c}$, M. PȨKąA $^{d}$, \\ H. SZYMCZAK ${ }^{c}$ AND A.I. VOITENKO ${ }^{b}$ \\ ${ }^{a}$ Hiroshima University, Faculty of Integrated Arts and Sciences \\ Higashi-Hiroshima, 739-8521, Japan \\ ${ }^{b}$ Institute of Physics, prospekt Nauki 46, 03028 Kiev, Ukraine \\ ${ }^{c}$ Institute of Physics, Polish Academy of Sciences \\ al. Lotników 32/46, PL-02-668 Warsaw, Poland \\ ${ }^{d}$ Department of Chemistry, University of Warsaw
}

al. Żwirki i Wigury 101, PL-02-089 Warsaw, Poland

\begin{abstract}
Differential conductance $G$ as a function of the bias voltage $V$ was measured for break-junctions of superconducting $\mathrm{Bi}_{2} \mathrm{Sr}_{2} \mathrm{CaCu}_{2} \mathrm{O}_{8+\delta}$ and $\mathrm{YBa}_{2} \mathrm{Cu}_{3} \mathrm{O}_{7-\delta}$. The dependences $G(V)$ for both materials clearly demonstrate the so-called dip-hump structures outside the gap region. A theory, which suggests the charge-density-wave origin of the dip-hump structures and explains its specific form by intrinsic inhomogeneity of cuprate materials, was developed. The well-known pseudogap features in the tunnel spectra of high- $T_{\mathrm{c}}$ oxides found both below and above the superconducting critical temperature are also described by the theory, which testifies that both the pseudogap and the dip-hump structures have the same origin. Competing theories and various $G(V)$ peculiarities found for a number of superconducting oxides are briefly discussed.
\end{abstract}

PACS numbers: 73.43.Jn, 71.45.Lr, 74.50.+r, 74.81.--g

\section{Introduction}

Pseudogap (PG) manifestations in tunneling spectroscopy, photoemission spectroscopy (ARPES), resistive and optical measurements of cuprates are well known [1], but still remain explained ambiguously. The main problem consists in the choice between a viewpoint that the PG comprises some kind of a precursor phenomenon tightly connected to superconductivity as such [2] and an alternative idea of a competition between the Cooper pair instability and some other collective state, such as conventional or unconventional charge- or spin-density waves (CDWs or SDWs), antiferromagnetism, stripe ordering, etc. [3]. Plenty of facts supporting 
the latter viewpoint are available, although the experimentum crucis has not been carried out yet. Really, phase diagrams for a number of cuprate systems show that PGs exist for underdoped compositions and gradually disappear with hole or electron doping, accompanied by a steep rise of the superconducting critical temperature, $T_{\mathrm{c}}[4-7]$. The subsequent decrease in $T_{\mathrm{c}}$ in the overdoping range seems to be unlinked to the PGs and might originate, e.g., from overscreening of the electron-phonon-interaction matrix elements [8], if cuprate superconductivity is due to this traditional mechanism, which seems quite plausible [9-11]. Moreover, PGs and superconducting gaps $\Delta$ (SGs) are frequently observed simultaneously, although having substantially different energy scales [7, 12, 13].

The dependences of the tunnel quasiparticle differential conductance $G=$ $\mathrm{d} J / \mathrm{d} V$ on the bias voltage $V$ (hereafter referred to as current-voltage characteristics, CVCs), where $J$ is the quasiparticle current, are especially important to discriminate between PGs and SGs [14], serving as local probes sensitive to any variation of the electron density of states (DOS) [1]. In particular, for cuprates, tunnel spectroscopy measurements clearly revealed, besides other peculiarities, several specific features, which - from our viewpoint - are interrelated and testify that pseudogapping in these objects is actually a manifestation of CDW existence. At low temperatures, $T$, it is difficult to identify CDW in the $\mathrm{SG}$ region $|e V|<\Delta$ ( $e>0$ being the elementary charge) against the SG background. Nevertheless, CDWs play a dominant role in the formation of the so-called dip-hump structures (DHSs). As for the range $T>T_{\mathrm{c}}$, the CDW-driven DOS depletion is very conspicuous (see description and discussion in Ref. [15]). Such consequences of the CDW formation in superconductors are not at all an exceptionally rare phenomenon. On the contrary, the hostile coexistence between CDWs and superconductivity has been observed in many materials [3, 16-20]. Unidirectional CDW-like modulations and checkerboard structures have been also found in a number of high- $T_{\mathrm{c}}$ oxides [21-23], which provides a direct support for our viewpoint. At the same time, to explain the smeared character of DHSs and PG-driven shallow DOS depletions, a wide spread of the PG parameters was assumed in close agreement with the observations [12, 21-24].

In this paper, we further develop the theory [15] of DHSs and PGs in CVCs of $s$-wave superconductors, making calculations not only for non-symmetrical $\mathrm{S}-\mathrm{I}-\mathrm{N}$ junctions (S stands for a superconductor, I for an insulator, and $\mathrm{N}$ for a normal metal), but also for symmetrical (S-I-S) ones. Moreover, we experimentally demonstrate the emergence of DHSs in break junctions (BJ) made of $\mathrm{Bi}_{2} \mathrm{Sr}_{2} \mathrm{CaCu}_{2} \mathrm{O}_{8+\delta}$ (BSCCO) single crystals and $\mathrm{YBa}_{2} \mathrm{Cu}_{3} \mathrm{O}_{7-\delta}(\mathrm{YBCO})$ polycrystalline crystals. It has been shown that the theoretical model makes it possible to fit experimental data adequately.

\section{Theoretical synopsis}

The comprehensive formulation of the self-consistent theory for a homogeneous partially gapped CDW superconductor (CDWS) and the analysis of the 
influence of CDWS inhomogeneity on the tunnel CVCs of junctions with such electrodes can be found elsewhere $[15,25]$. The theory demonstrates that a tunnel junction with a homogeneous CDWS as an electrode is sensitive to the CDW phase $\varphi$ (but not to the superconducting one), so that the resulting CVC can be non-symmetric. The CVC features are the functions of the CDWS parameters, such as the "bare" superconducting and dielectric order parameters (OPs) $\left(\Delta_{0}^{*}\right.$ and $\tilde{\Sigma}_{0}^{*}=\Sigma_{0}^{*} \mathrm{e}^{\mathrm{i} \varphi}$, respectively), and the degree of dielectric gapping of the Fermi surface (FS) $\mu$, as well as on the temperature $T$. The dispersion of each of the CDWS parameters gives rise to the smearing of the CVC features, and, in paper [15], such a theoretical consideration for a non-symmetric CDWS-I-N junction was made. Here, we would like to confront the results of calculations with experimetal ones for both symmetric and non-symmetric tunnel configurations.

It should be indicated that even in the actual experimental situation, when it is difficult to distinguish between PGs (i.e. CDW gaps) and SGs, the higher inhomogeneity of the former was noticed while analyzing the scanning tunnel microscopy (STM) data for BSCCO [22] (see also Ref. [23]). An ingenious renormalization method, used to process the results of STM measurements for an overdoped $\left(\mathrm{Bi}_{1-y} \mathrm{~Pb}_{y}\right)_{2} \mathrm{Sr}_{2} \mathrm{CuO}_{6+x}$ sample with $T_{\mathrm{c}}=15 \mathrm{~K}$ [12], allowed it to be seen more or less directly.

Every averaged parameter $G_{0}^{*}$ (it can be $\Sigma_{0}^{*}$ or $\Delta_{0}^{*}$, or both) was regarded distributed within the finite interval $\left[G_{0}^{*}-\delta G_{0}^{*}, G_{0}^{*}+\delta G_{0}^{*}\right]$ according to a bell-shaped distribution function $W(x)$ (see discussion in Ref. [15]). In any case, the specific form of $W(x)$ is not crucial for final results and conclusions.

We assume that both $\Delta$ and $\Sigma$ are $s$-wave OPs. Nevertheless, our approach is also applicable to superconductors with the $d$-wave symmetry, which is usually considered true for cuprates. In any case, taking the $d$-wave symmetry into account would result in a more adequate description of the CVC section near $V=0$, whereas both DHS and PG features are located beyond the SG region.

The following question arises: Can SDWs bring about a similar effect? The answer is: Yes, they can, because tunnel junctions with SDW-superconductors should also be sensitive to the SDW phase [26]. However, the emergence of SDWs is not accompanied by charge redistribution. At the same time, such a charge modulation does take place in high- $T_{\mathrm{c}}$ oxides (see, e.g., Refs. [23, 27]), which explains our choice in favor of the CDW scenario. Moreover, in hole-doped cuprates, the antiferromagnetic region of the phase diagram lies far away from the superconducting dome $[4,5]$, contrary to that, which exhibits the PG phenomena, making the SDW scenario less probable.

\section{Experimental results and fitting}

The conductances $G$ at low $T$ of our BJs for a single BSCCO crystal and a polycrystalline YBCO sample are shown in Figs. 1 and 2, respectively. As one can see, the DHSs are very pronounced. On the basis of our theory, we fitted 


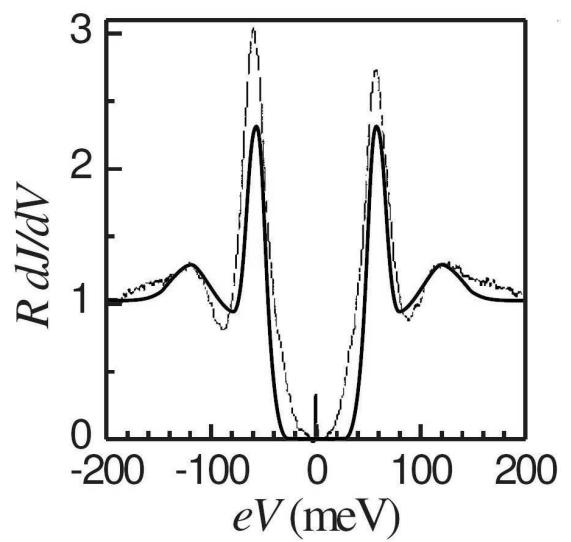

Fig. 1. Normalized experimental differential conductance $G=\mathrm{d} J / \mathrm{d} V$ for a BSCCO break junction (thin curve) measured at $T=4.2 \mathrm{~K}$, where $J$ is the quasiparticle tunnel current and $V$ the bias voltage across the junction, versus the $e V$ value, where $e$ is the elementary charge. The amplitude of voltage modulation $\delta V$ for calculating $G$ was $1 \mathrm{meV}$. The bold curve corresponds to the calculated $e V$-dependence of the dimensionless differential conductance $R G$ of a tunnel junction between two identical inhomogeneous CDW superconductors. Here, $R$ is the resistance of the junction in the normal state. The parameters of the CDW superconductors are $\Delta_{0}^{*}=30 \pm 15 \mathrm{meV}, \Sigma_{0}^{*}=90 \pm 35 \mathrm{meV}$, the FS CDW-gapping parameter $\mu=0.08$, and the temperature $T=4.2 \mathrm{~K}$. The interval of numerical differentiantion $\delta V=1 \mathrm{meV}$.

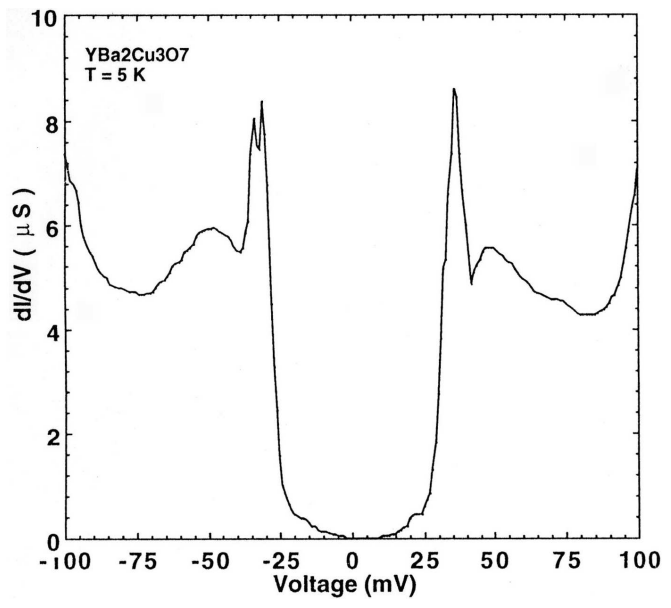

Fig. 2. Experimental differential conductance for an YBCO break junction.

the experimental curve (the bold curve in Fig. 1). The agreement is quite good and the required values of the problem parameters seem realistic. In particular, the value of the control parameter $\mu$ turned out rather small, which is compatible 
with observed subdominant overall CDW structures on the crystal surfaces. On the other hand, tunnel spectroscopy works as an amplifier and clearly detects both kinds of CDW-driven features (DHSs and PGs).

\section{Results of high- $T$ calculations}

The results of calculations also demonstrate that the same "CDW + inhomogeneity" combination explains well the PG phenomena at high temperatures, when the DHS becomes smoothed out. An example of the transformation of the DHS-decorated tunnel spectra into the typical PG-like ones is shown in Fig. 3 for CDWS-I-N junctions with $\varphi=\pi$ (part (a)) and $\pi / 2$ (part (b)). The CDWS parameters are $\Delta_{0}^{*}=20 \mathrm{meV}, \Sigma_{0}^{*}=50 \mathrm{meV}, \mu=0.1$, and $\delta \Sigma_{0}^{*}=20 \mathrm{meV}$. For this parameter set, the "actual" superconducting critical temperatures $T_{\mathrm{c}}$ of CDWS domains lie within the interval 114-126 K, and the temperatures of the CDW phase transition $T_{\mathrm{d}}$ are in the range $197-461 \mathrm{~K}$. This figure makes lucid the transformation of CVC from the DHS-including pattern at $T$ well below $T_{\mathrm{c}}$ into the PG-like ones in the vicinity of $T_{\mathrm{c}}$ or above it. The asymmetric curves displayed in part (a) are similar to the measured STM $G^{\mathrm{ns}}(V)$ dependences for overdoped and underdoped BSCCO compositions [24].
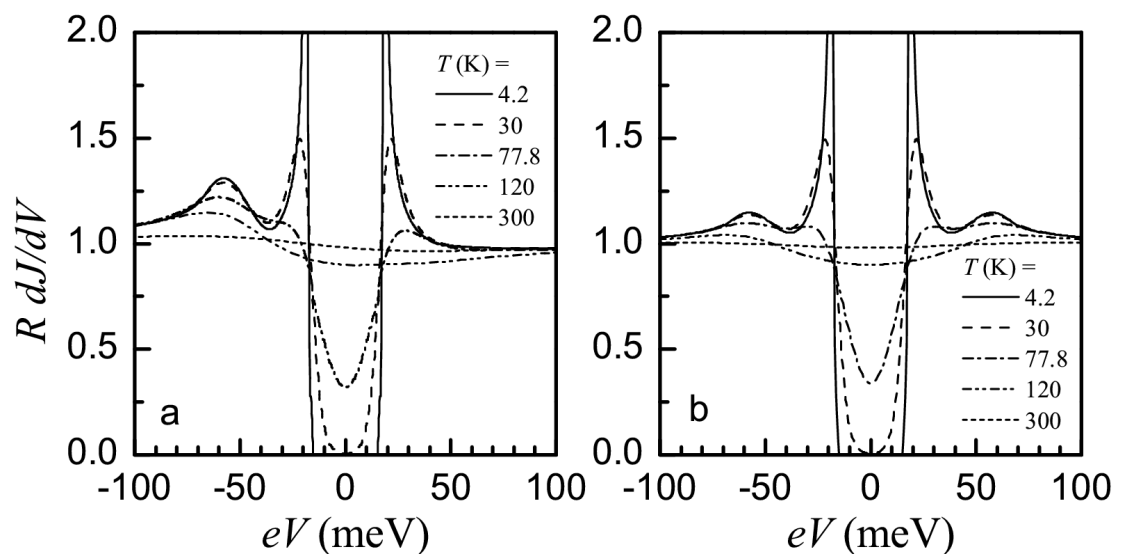

Fig. 3. $G(V)$-dependences of the tunnel junction between an inhomogeneous CDWS and a normal metal for various temperatures $T$. The dielectric OP phase $\varphi=\pi$ (part (a)) and $\pi / 2$ (part (b)), and the dielectric OP-amplitude spread $\delta \Sigma_{0}^{*}=20 \mathrm{meV}$. All other parameters are indicated in the text.

Similar CDW-related features should be observed in the CVCs measured for symmetric CDWS-I-CDWS junctions. The $G(V)$ dependences for this case with the same set of parameters as in Fig. 3 are shown in Fig. 4. As is readily seen, the transformation of the symmetric DHS pattern into the PG-like picture is similar to that for the non-symmetric junction. This simplicity is caused by the small parameter $\mu=0.1$, so that the features at $e V= \pm 2 D$, which are proportional 
to $\mu^{2}$, are inconspicuous on a chosen scale. The value of the order of 0.1 for $\mu$ was chosen to approximate the experimental data for BSCCO by theoretical CVCs. Raman investigations [28] for the oxide $\mathrm{La}_{2-x} \mathrm{Sr}_{x} \mathrm{CuO}_{4}$, which possesses the maximal $T_{\mathrm{c}} \approx 42 \mathrm{~K}$ at $x=0.15$, also demonstrate that this parameter is equal to about 0.15 .

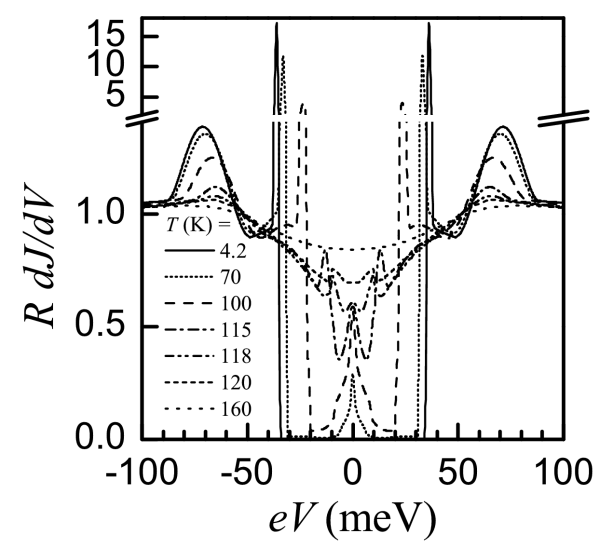

Fig. 4. The same as in Fig. 3a, but for a symmetric CDWS-CDWS junction.

The appearance of the $T$-driven zero-bias peaks is a salient feature of certain CVCs displayed in Fig. 4. As is well known, this peak is caused by tunneling of thermally excited quasiparticles between empty states with an enhanced DOS located above and below equal SGs in symmetric S-I-S junctions. The experimenter should be careful not to confuse this peak with the dc Josephson peak restricted to $V=0$, which is often seen for symmetric high- $T_{\mathrm{c}}$ junctions [14]. The distinction consists in the growth of the quasiparticle zero-bias maximum with $T$ up to a certain temperature, followed by its drastic reduction. On the other hand, the Josephson peak decreases monotonously as $T \rightarrow T_{\mathrm{c}}$.

\section{Conclusions}

We have shown that CDW manifestations can explain both subtle DHSs in the tunnel spectra for high- $T_{\mathrm{c}}$ oxides and large PG features observed both below and above $T_{\mathrm{c}}$. The DHS is gradually transformed into the PG-like DOS depletion as $T$ grows. Hence, both phenomena are closely interrelated, being the manifestations of the same CDW-governed feature smeared by CDWS inhomogeneity. Therefore, one should not try to explain DHSs and PGs separately. The dependences of the calculated CVCs on the CDW phase $\varphi$ fairly well describe the asymmetrical features of the tunnel spectra for BSCCO and related compounds. 


\section{Acknowledgments}

A.M.G. and A.I.V. are grateful to Kasa im. Józefa Mianowskiego, Polski Naftowy Koncern ORLEN, and Fundacja Zygmunta Zaleskiego for the financial support of their visits to Warsaw. A.M.G. is also grateful to the Japan Society for the Promotion of Science (FY2007 JSPS Fellowship Program, grant No. S-07042) for the invitation to Hiroshima University and the grant given in the framework of the Visitors Program of the Max Planck Institute for the Physics of Complex Systems (Dresden, Germany). M.S.L. was supported by the Ministry of Science and Informatics in Poland (grant No 202-204-234). T.E. was supported by JSPS Grant-in-Aid for Scientific Research (No. 19540370). We thank Prof. Jun Akimitsu (Tokyo) as well as Dr. Yasuhiro Asano, Dr. Koichi Ichimura, Dr. Katsuhiko Inagaki, Dr. Toru Matsuura, Prof. Migaku Oda, and Prof. Satoshi Tanda (Sapporo) for very useful discussions.

\section{References}

[1] Ø. Fischer, M. Kugler, I. Maggio-Aprile, C. Berthod, Rev. Mod. Phys. 79, 353 (2007).

[2] S. Chakravarty, R.B. Laughlin, D. Morr, C. Nayak, Phys. Rev. B 63, 094503 (2001).

[3] A.M. Gabovich, A.I. Voitenko, M. Ausloos, Phys. Rep. 367, 583 (2002).

[4] T. Nakano, N. Momono, M. Oda, M. Ido, J. Phys. Soc. Jpn. 67, 2622 (1998).

[5] R.M. Dipasupil, M. Oda, N. Momono, M. Ido, J.Phys. Soc. Jpn. 71, 1535 (2002).

[6] L. Alff, Y. Krockenberger, B. Welter, M. Schonecke, R. Gross, D. Manske, M. Naito, Nature 422, 698 (2003).

[7] M.L. Tacon, A. Sacuto, A. Georges, G. Kotliar, Y. Gallais, D. Colson, A. Forget, Nature Phys. 2, 537 (2006).

[8] A.M. Gabovich, Fiz. Tverd. Tela 22, 3231 (1980).

[9] A. Lanzara, P.V. Bogdanov, X.J. Zhou, S.A. Kellar, D.L. Feng, E.D. Lu, T. Yoshida, H. Eisaki, A. Fujimori, K. Kishio, J.-I. Shimoyama, T. Noda, S. Uchida, Z. Hussain, Z.X. Shen, Nature 412, 510 (2001).

[10] K.M. Shen, F. Ronning, D.H. Lu, F. Baumberger, N.J.C. Ingle, W.S. Lee, W. Meevasana, Y. Kohsaka, M. Azuma, M. Takano, H. Takagi, Z.-X. Shen, Science 307, 901 (2005).

[11] G.-M. Zhao, Phys. Rev. B 71, 104517 (2005).

[12] M.C. Boyer, W.D. Wise, K. Chatterjee, M. Yi, T. Kondo, T. Takeuchi, H. Ikuta, E.W. Hudson, Nature Phys. 3, 802 (2007).

[13] W.S. Lee, I.M. Vishik, K. Tanaka, D.H. Lu, T. Sasagawa, N. Nagaosa, T.P. Devereaux, Z. Hussain, Z.-X. Shen, Nature 450, 81 (2007).

[14] T. Ekino, Y. Sezaki, H. Fujii, Phys. Rev. B 60, 6916 (1999).

[15] A.M. Gabovich, A.I. Voitenko, Phys. Rev. B 75, 064516 (2007).

[16] D. Andres, M.V. Kartsovnik, W. Biberacher, K. Neumaier, E. Schuberth, H. Müller, Phys. Rev. B 72, 174513 (2005). 
[17] T. Sakamoto, M. Wakeshima, Y. Hinatsu, K. Matsuhira, Phys. Rev. B 75, 060503 (2007).

[18] T. Kiss, T. Yokoya, A. Chainani, S. Shin, T. Hanaguri, M. Nohara, H. Takagi, Nature Phys. 3, 720 (2007).

[19] O. Degtyareva, M.V. Magnitskaya, J. Kohanoff, G. Profeta, S. Scandolo, M. Hanfland, M.I. McMahon, E. Gregoryanz, Phys. Rev. Lett. 99, 155505 (2007).

[20] T. Dobashi, T. Sato, S. Souma, T. Takahashi, H. Kaneko, Y. Ishihara, H. Okamoto, Phys. Rev. B 76, 045121 (2007).

[21] J. Lee, K. Fujita, K. McElroy, J.A. Slezak, M. Wang, Y. Aiura, H. Bando, M. Ishikado, T. Masui, J.-X. Zhu, A.V. Balatsky, H. Eisaki, S. Uchida, J.C. Davis, Nature 442, 546 (2006).

[22] A. Hashimoto, N. Momono, M. Oda, M. Ido, Phys. Rev. B 74, 064508 (2006).

[23] Y.H. Liu, K. Takeyama, T. Kurosawa, N. Momono, M. Oda, M. Ido, Phys. Rev. B 75, 212507 (2007).

[24] K.K. Gomes, A.N. Pasupathy, A. Pushp, S. Ono, Y. Ando, A. Yazdani, Nature 447, 569 (2007).

[25] A.M. Gabovich, M.S. Li, H. Szymczak, A.I. Voitenko, J. Phys., Condens. Matter 15, 2745 (2003).

[26] A.M. Gabovich, A.I. Voitenko, Phys. Rev. B 60, 14897 (1999).

[27] T. Hanaguri, C. Lupien, Y. Kohsaka, D.-H. Lee, M. Azuma, M. Takano, H. Takagi, J.C. Davis, Nature 430, 1001 (2004).

[28] S. Sugai, Y. Takayanagi, N. Hayamizu, Phys. Rev. Lett. 96, 137003 (2006). 